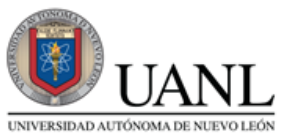

FACPYA
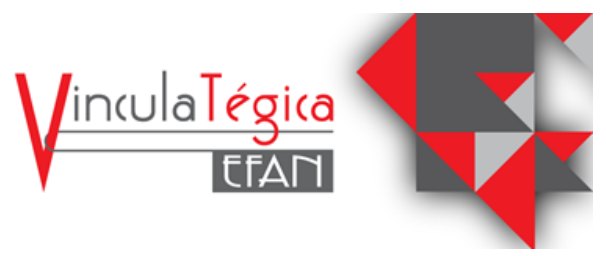

\title{
Capacitación laboral en herramientas digitales
}

\author{
Laura Alicia Hernández-Morenoํㅜ Ma. de Lourdes Hernández-Saldaña ${ }^{2}$ y María Teresa \\ Tovar-Morales ${ }^{3}$
}

${ }^{1}$ Universidad Autónoma de Nuevo León; Facultad de Contaduría Pública y Administración, laura.hernandezmr@uanl.edu.mx, Ciudad Universitaria San Nicolás de los Garza, Nuevo León, México. C.P. 66451., Teléfono (81) 8329-4001

${ }^{2}$ Universidad Autónoma de Nuevo León; Facultad de Contaduría Pública y Administración, lourdes.hernandezsld@uanl.edu.mx, Ciudad Universitaria San Nicolás de los Garza, Nuevo León, México.

C.P. 66451., Teléfono (81) 8329-4001

${ }^{3}$ Universidad Autónoma de Nuevo León; Facultad de Contaduría Pública y Administración, maria.tovarmrl@uanl.edu.mx, Ciudad Universitaria San Nicolás de los Garza, Nuevo León, México. C.P. 66451., Teléfono (81) 8329-4001

Información del artículo revisado por pares

Fecha de aceptación: junio-2021

Fecha de publicación en línea: diciembre-2021

DOI: https://doi.org/10.29105/vtga7.2-74

\section{Resumen}

Los cambios tecnológicos han originado que las personas se capaciten y realicen de forma diferente su trabajo adoptando las nuevas tecnologías, sin embargo, la capacitación no solo concierne al trabajador sino también a los empleadores e instituciones educativas o quienes se encargan de capacitar; todos ellos tienen la obligación de cubrir los perfiles profesionales que demande el mercado laboral. El estudio tuvo como objetivo determinar si los estudiantes de maestría de una universidad pública del noreste de México se están capacitando o no en herramientas digitales. La metodología se definió como alcance de tipo exploratorio descriptivo, se creó y aplicó una encuesta integrada por 16 preguntas cerradas y 3 abiertas. La muestra fue no probabilística integrada por 550 estudiantes. Los resultados muestran una disminución de capacitación del $24.2 \%$ durante el año 2020 respecto al 2019 y esto no es debido a un problema de desempleo originado por la pandemia COVID19 , además se identifica que el $41.5 \%$ presentan una capacitación o ninguna al año. El porcentaje más alto de capacitación se da en las herramientas básicas de Microsoft Office con un 30\% (Word,

\begin{abstract}
Technological changes have caused people to train and perform their work differently by adopting new technologies, however, training does not only concern the worker but also the employers and educational institutions or those who are in charge of training; all of them should cover the professional profiles demanded by the labor market. The study aimed to determine whether or not master's students from a public university in northeastern Mexico are being trained in digital tools. The methodology was defined as a descriptive exploratory scope, a survey consisting of 16 closed questions and 3 open questions was created and applied. The sample was non-probabilistic, made up of 550 students. The results show a $24.2 \%$ decrease in training during 2020 compared to 2019 and this is not due to an unemployment problem caused by the COVID-19 pandemic, it is also identified that $41.5 \%$ present training or none per year. The highest percentage of training is given in the basic Microsoft Office tools with $30 \%$ (Word, Excel, and PowerPoint), followed by specialized packages with $28.2 \%$, such as SPSS, Contpaqi, Aspel SAE, ERP SAP, Photoshop, among others. Universities, corporations, and governments must create an alliance to significantly increase the
\end{abstract}


Excel y PowerPoint), seguida de los paquetes especializados con un $28.2 \%$, tales como, SPSS, Contpaqi, Aspel SAE, ERP SAP, Photoshop, entre otros. Es importante que universidades, corporaciones y gobiernos generen una alianza a fin de aumentar significativamente el porcentaje de capacitación a fin de satisfacer las necesidades del mercado laboral, así como analizar el costobeneficio que se puede dar de una capacitación presencial a una en línea.

Palabras clave: capacitación laboral, herramientas digitales, capacitación presencial o en línea, pandemia covid-19.

\section{INTRODUCCIÓN}

Los cambios que ha provocado la globalización, como los sociales, así como el surgimiento y adaptación de nuevas tecnologías, han traído consigo la innovación o formas diferentes de realizar el trabajo; por lo cual se considera que el trabajador deberá de actualizar o desarrollar nuevos conocimientos, competencias y habilidades que le permitan integrarse a los cambios que demandan las empresas. En la actualidad las competencias digitales son las de mayor interés; sin embargo, el desarrollo de las competencias, es un aspecto que no solo concierne al trabajador, sino también al gobierno, a las empresas y empleadores y a las instituciones educativas $\mathrm{o}$ de capacitación.

De acuerdo con la Organización Internacional del Trabajo (OIT) cada uno de los involucrados en el desarrollo de las competencias digitales juega un papel importante. Respecto al gobierno, señala que deberá de establecer y adoptar políticas que permitan fomentar el desarrollo de las competencias digitales que se requieran en las empresas. Por el lado, de las empresas y empleadores señala que, con el objetivo de lograr un crecimiento económico, se deberá invertir en la capacitación de los trabajadores a fin de que estos continúen con una activa participación en su enseñanza aprendizaje y en la readaptación profesional que los tiempos demanden. Sobre los sistemas de educación y formación; refiere la necesidad de aprovechar las nuevas tecnologías educativas y enfocar la atención a las percentage of training to meet the needs of the labor market, as well as analyze the cost-benefit that can be given from face-to-face training to an online one.

Keywords: job training, digital tools, face-to-face or online training, covid-19 pandemic

JEL: E24, J24, J41.

competencias digitales. Finalmente, sobre los trabajadores indica que, si es del interés de estos el mantener o conseguir un trabajo, deberán de continuar de forma permanente con su enseñanza aprendizaje a fin de actualizar su conocimiento, competencias y habilidades o para adquirir nuevas (ILO, 2021).

De acuerdo con estudios realizados por importantes compañías internacionales en reclutamiento de personal tales como Manpower Group (2020), Hays (2019) y Adecco señalado por el financiero (2019) y el Universal (2020), dentro de los aspectos que describen en sus estudios, refieren problemas de escasez de talento o falta de personal capacitado en áreas técnicas y en otras más. Además, se presenta el problema de que las empresas o empleadores no están cumpliendo con la capacitación hacia sus empleados. Por otro lado, se señala la importancia de que las universidades y las organizaciones capaciten a sus estudiantes y empleados respectivamente, a fin de que estos puedan contar con las habilidades digitales para poder satisfacer la necesidad del mercado. Se sugiere el desarrollo interno del talento con el propósito de evitar la migración de empleados, al promover en ellos perfiles híbridos en los cuales puedan realizar varias funciones, aumenten su productividad y por consiguiente se vuelvan más eficientes.

Si bien el aprendizaje y capacitación hoy en día ya no se lleva a cabo solo en un espacio físico dentro de la organización, este proceso ha evolucionado a un ambiente en 
línea. En acuerdo a lo indicado por Orri (2018), actualmente algunas universidades y empresas han ido incursionando en capacitaciones en línea a través de cursos conocidos como MOOC por sus siglas en ingles "Masive Open Online Course" que en idioma castellano significa Cursos en Línea Masivos y Abiertos. Estos se tratan de cursos abiertos y gratuitos o de paga, si se requiere obtener un certificado oficial; se hace uso de plataformas reconocidas mundialmente como Coursera, EdX, Udemy, entre otras más, que integran materiales basados por lo general en videos cortos lo que permite una modalidad de estudio flexible, es decir, que la persona a su ritmo y a su tiempo pueda ir avanzando en el contenido del curso. Una cuestión importante por resaltar (para llevar un curso de este tipo) es que aparte de tener que contar con habilidades técnicas, se requiere que la persona tenga la habilidad de aprender por si misma o de ser autodidacta. La revista expansión (2020) indica que la necesidad de capacitación y aislamiento derivados por la pandemia COVID-19 originaron un incremento en las inscripciones hacia estas plataformas, tal es el caso de la plataforma Udemy en donde se reporta un incremento del $114 \%$ en las inscripciones en México.

$$
\text { Considerando los aspectos }
$$

previamente descritos, el presente estudio tiene como objetivo determinar si los estudiantes de maestría de una universidad pública del noreste de México se están capacitando o no en herramientas digitales. El estudio es justificable y viable, en primera instancia, se enfoca a un tema actual y en el cual los sistemas educativos están incluidos; posteriormente, la naturaleza de los estudiantes, los cuales son en su mayoría trabajadores activos y finalmente, esta investigación (en conjunto con otras) permite determinar la administración académica que habrá de realizarse, en un curso de tecnologías que se impartirá a los estudiantes que participan en el estudio. Las preguntas de investigación que rigen este trabajo son las siguientes: ¿Cuántos estudiantes tuvieron al menos una capacitación durante el año 2019 sobre herramientas digitales? ¿Debido a la pandemia, ha sido mayor el número de capacitaciones sobre herramientas digitales? ¿Las empresas incentivan o apoyan a sus trabajadores para su capacitación en herramientas digitales? ¿Invierten los trabajadores en su capacitación sobre herramientas digitales? ¿Cuáles son las herramientas digitales más actuales sobre las que se han capacitado los trabajadores? ¿Son los trabajadores enfocados al área de tecnologías, quienes más se capacitan en herramientas digitales? ¿Se hace uso de los MOOC para capacitarse? ¿Son los trabajadores de más de 50 años de edad los que menos se capacitan en herramientas digitales? ¿Existen trabajadores que tienen niveles de conocimiento bajo en las herramientas básicas de Microsoft Office tales como Word, Excel y PowerPoint?

\section{MARCO TEÓRICO}

Chiavenato (2020) señala, cuando se refiere a gestión del talento humano, que no se trata de la gestión de las personas en sí, sino los talentos y competencias que ellas aprenden, poseen y aplican. Además, no solamente invierten en los productos, servicios, y en lo clientes; sino en las personas que saben cómo satisfacerlos, de este modo se convierten en el elemento básico del éxito de la empresa.

Por otro lado, (Werther et al.,2019) el concepto de capital intelectual hace alusión a la suma de todos los conocimientos con los que cuenta los empleados y que a su vez representan una ventaja competitiva para la organización; ya que son los hombres y las mujeres, quienes, a través de prácticas innovadoras, participan en la toma de decisiones.

Werther et al. (2019) señalan que, en relación con el capital humano, que el principal desafío durante el siglo XXI continúa siendo la reciprocidad entre los diversos elementos como las personas, organizaciones, economías nacionales y de manera particular las tecnologías de la información (TI), la internet y las redes sociales; ya que han orillado a las organizaciones a replantear formas de organización y funciones.

Respecto al desarrollo de talentos 
Chiavenato (2019) ha señalado los siguiente:

Los procesos de desarrollo de recursos humanos incluyen las actividades de capacitación, desarrollo de personal y desarrollo organizacional; todas ellas representan las inversiones de la organización en su personal en el sentido de incrementar el aprendizaje organizacional y el conocimiento corporativo. (p. 324)

Respecto al término aprendizaje (Chiavenato,2019) es el proceso a través del cual las personas, en el transcurso de su vida, adquieren conocimiento tanto de su medio como de sus relaciones; es por ello que ocurre cuando se manifiesta un cambio en la conducta de la persona como resultado de una respuesta previa. Es precisamente el aprendizaje que día con día nos vuelve mejores; además estimula los cambios a nivel individual $y$ el organizacional.

Para clarificar y diferenciar los términos de capacitación y desarrollo se presenta lo señalado por Werther et al. (2019):

La capacitación es el desarrollo de conocimientos, habilidades y técnicas operativas y administrativas de todos los niveles de personal auxiliar a los miembros de la organización para desempeñar su trabajo actual.

Se entiende por desarrollo los programas dirigidos a empleados de niveles medios y superiores, a corto, mediano y largo plazos a quienes se les da una preparación que les servirá para el futuro. (p. 170)

Chiavenato (2020) presenta este otro concepto de capacitación, primero en su forma tradicional como: el proceso a través del cual se prepara a la persona con la finalidad de que pueda desempeñar de forma muy satisfactoria las tareas específicas para el puesto que ocupa y posteriormente, desde un enfoque más actual, señala que es un medio que involucra el desarrollo de las competencias de las personas para contribuir al logro de los objetivos organizacionales y a su vez generar valor.

En relación a la comparativa entre los métodos de capacitación tradicionales y cómo se imponen las nuevas técnicas, Chiavenato (2019) menciona que la tecnología informática ha repercutido en la disminución de los costos de operación y menciona las siguientes: recursos audiovisuales (grabaciones que se distribuyen de forma diversa), teleconferencias (reuniones en las que se emplea audio y video), comunicaciones electrónicas (comunicación interactiva dentro de la red de la organización), correo electrónico (redes de computadoras internet o intranet) y tecnología multimedia (voz, video texto).

Siguiendo esta misma línea, respecto a contrastar métodos tradicionales con otros más contemporáneos, Werther et al. (2019), describen a la capacitación en línea como una gran innovación en este campo; particularmente los sistemas computarizados de administración de aprendizaje o LMS por su sigla en idioma inglés (Learning Management Systems) del cual menciona ventajas como, las de mantener registros actuales de los conocimientos adquiridos y permitir que se cuente con una guía y control respecto a los avances obtenidos.

Un elemento muy importante con respecto de la capacitación en México está determinado por la reglamentación legal que obliga a su impartición; ello corresponde a la Carta Magna o Constitución Política de los Estados Unidos Mexicanos en el artículo 123 constitucional, en el apartado A, fracción XIII, en donde señala que:

"Las empresas estarán obligadas a proporcionar a todos sus trabajadores, capacitación o adiestramiento para el trabajo y para efecto inscribe que la ley reglamentaria determinará los sistemas, métodos y procedimientos conforme a los cuales los patrones deberán cumplir con dicha obligación”. (p.137)

De manera particular la Ley Federal del Trabajo señala en el Capítulo III BIS, De la Productividad, Formación y Capacitación de los Trabajadores en el Artículo 153-A lo siguiente:

"Los patrones tienen la obligación de proporcionar a todos los trabajadores, y éstos a recibir, la capacitación o el adiestramiento en su trabajo que le permita elevar su nivel de vida, su competencia laboral y su productividad, conforme a los planes y programas formulados, de común acuerdo, por 
el patrón y el sindicato o la mayoría de sus trabajadores". (p.43)

De acuerdo a la revista de negocios a nivel mundial Forbes, en su edición mexicana emitida a través de la Internet en el mes de enero del 2020, señala que Online Carrer Center mejor conocido como OCC Mundial,y presenta en su sitio una serie de informaciones y consejos para las personas que buscan empleo (ya que se considera una de las bolsas de trabajo más grandes en México) y hace mención a interesantes cifras estadísticas relacionadas con el tema de capacitación; en ellas se muestra que únicamente el $11 \%$ de las empresas la proporcionaron a sus empleados durante el año 2019 y que disminuyó un 21\% respecto de un año antes. Además refiere que, para ese mismo año 2019, creció de un $8 \%$ a un $15 \%$ la autocapacitación en línea. Las áreas más destacadas en las que se actualizaron conocimientos fueron en: idiomas, derecho, contabilidad, administración e ingeniería.

Asimismo, la empresa consultora Hays México, que es líder a nivel global en el ramo de reclutamiento especializado, ha señalado en su reporte del año 2019, que se presentan aparentes incongruencias respecto al hecho de que el $44 \%$ de las empresas mexicanas afirmaban haber generado capacitación a sus empleados, mientras que los colaboradores de las mismas solo el $27 \%$ identifican haber obtenido dichos beneficios. Desde la óptica de los empleadores, han considerado la capacitación como fundamental para el crecimiento de las empresas, el $44 \%$ refiere que, al momento de reclutar personal, la falta de especialización es el factor que mayor dificultad representa.

El Gobierno de México a través de la Secretaría del Trabajo y Previsión Social (STPS) presenta el portal Concertación y capacitación laboral considerado como el primer sitio web para esa especialización y proporcionado de forma gratuita; su finalidad es proveer información y herramientas para cubrir el objetivo de aumentar la productividad de los trabajadores. En él se afirma que, para todo patrón, la capacitación y adiestramiento es una obligación constitucional (como ya se mencionó en párrafos anteriores). En dicho portal se pueden consultar temas de interés como lo son: capacitación y adiestramiento, estudios para la capacitación, productividad laboral, estadísticas y reforma laboral. De ese mismo portal se vincula el Programa para Capacitación a Distancia para Trabajadores (PROCADIST) mismo que se promueve como una plataforma educativa gratuita a través de internet, diseñado para que planeen su propio proceso de aprendizaje con el propósito de desarrollar o perfeccionar competencias y habilidades laborales.

De acuerdo con Hays México en su Reporte Laboral México de año 2019 se señalan cuatro razones por las cuales invertir en la capacitación laboral: la flexibilidad en los trabajos en miras de retener el talento humano, es decir reducir la rotación; promover la especialización a través del impulso de la tecnología con herramientas digitales. En relación con las políticas salariales, que sean revisadas con respecto a la competitividad, acorde a las demandas de los profesionistas; por último, no perder de vista la diversidad laboral, para la integración de las perspectivas presentadas por todos los grupos representativos.

Siguiendo con lo informado por PR Newswire al referirse al Reporte Laboral México 2019, señala el siguiente dato estadístico, en donde en 6 de cada 10 empresas en México no se lleva acabo inversión en capacitación para empleados; además, entre los resultados del estudio de forma particular, aborda el tema relacionado con la transformación digital y señala la utilización por parte de los empleados de plataformas tecnológicas para reducir las obligaciones administrativas; pues de acuerdo al éste el $25 \%$ refiere la creación de nuevas posiciones más especializadas con las que se integran talentos que presentan un perfil con el que se logran cotizar de forma más elevada en el mercado laboral.

MOOC es un acrónimo en inglés formado por las palabras Masive Open Online Course que en idioma castellano significa Cursos en Línea Masivos y Abiertos, de una manera simple se les considera como una forma evolutiva de la educación abierta en internet; trae consigo una filosofía inmersa 
que se relaciona con la liberación del conocimiento, de modo que pueda ser accesible a un amplio público. De forma general, estos cursos son impartidos por instituciones de cierta solvencia o acreditadas por medio de una plataforma educativa y que se vinculan con áreas de conocimiento específicas. La Universitat Jaume I refiere que, para que pueda ser considerado a distancia, requiere el cumplimiento de estos aspectos: "debe tener una estructura orientada al aprendizaje, con pruebas o evaluaciones que acreditarán el conocimiento adquirido, no tener limitación en las matriculaciones, poder ser seguido online, tener un carácter abierto y gratuito, con materiales accesibles de forma gratuita" (UJI,2021).

Recientemente los MOOC han incursionado en una gran variedad de temas, de ellos una cantidad importante son aplicables en las empresas; una limitante es que la mayoría no se encuentran disponibles en idioma español. La particularidad de estos cursos radica en que las organizaciones pueden, de forma individualizada, generar un plan formativo para cada empleado y de ese modo optimizar su aprovechamiento; se consideran una herramienta apropiada para una formación dinámica y lúdica, que puede ser empleada de forma complementaria a lo ya establecido de forma presencial. Algunas empresas han optado por elaborar varios MOOC de tipo empresarial en una plataforma online y han hecho mención que la inversión realizada ha sido una alternativa apropiada; otras más de diversos tamaños estiman o proyectan beneficios con respecto a la utilización de estos cursos para la formación de sus empleados.

La revista electrónica American Learning Media muestra de forma particular, un informe especial acerca de los MOOCs (Massive Open Online Courses) y proporciona un extracto que muestra una compilación de artículos con una diversidad temática para el desarrollo de cursos, diversidad de plataformas, opciones de proveedores, variedad de buscadores y hasta directorios de lo que han denominado "el universo MOOC". En él se puede apreciar una breve visión holística de cómo estos recursos han generado un impacto en la actualidad, ya que hace referencia a un mercado de tipo mundial y también latinoamericano; se abordan estudios de mercado, tasas de crecimiento, así como los temas más solicitados o buscados. De acuerdo con el artículo: Mundo MOOC: actualidad, buscadores y plataformas, publicado en el mes de agosto de 2016, menciona que el buscador Class Central (el cual proporciona una lista exhaustiva de cursos gratuitos en línea) señala que la cantidad total de estudiantes había superado los 35 millones de estudiantes y se encontraban impartiendo diversidad de cursos, en un total de 570 universidades. De esta misma fuente se señalan que el $75 \%$ son cursos en idioma inglés y que, de la diversidad de 16 lenguas incluidas, destacan el español y el francés. Otro dato que cabe señalar es que, de una gráfica de distribución de 11 temáticas, la de negocios y administración predomina por ser la más alta con un $16.8 \%$.

De este mismo informe especial del cual se hace referencia en el párrafo anterior, en el artículo titulado: 32 plataformas y proveedores MOOC se hace alusión a un listado de organizaciones que presentan sus proyectos y a su vez lo que les da soporte. Se trata de plataformas educativas o de e-learning, que refieren estar asociadas con universidades y organizaciones de todo el mundo; las cuales presentan una diversidad de lineamientos claves, señalan cantidad de instituciones educativas, países y usuarios o alumnos. Algunas mencionan, de forma muy específica, algún tipo de especialidad o tema; otras se promueven como gratuitas, o de bajo costo o bien con fines de lucro. Algunas cubren objetivos de tipo académico, o bien también integran el desarrollo profesional, otras más promueven la capacitación para las habilidades en el lugar de trabajo. De forma distintiva se hace alusión a la plataforma México X, la cual se promueve como una iniciativa gratuita de la Secretaría de Educación Pública del Gobierno de México, la cual reúne alrededor de 25 universidades e instituciones de educación superior, Secretarías de Estado, fundaciones, etc.; los cursos son de muy diversas temáticas, para este caso se hace hincapié en la fundación Televisa que promueve actualmente sus cursos 
de Herramientas digitales para la empleabilidad en sus versiones: básicas, intermedias y avanzadas (abril a diciembre del presente año). Se hace la aclaración de que no se ha logrado identificar un dato reciente de la cantidad de participantes en los que ha impactado; en cuanto a la cantidad de cursos que actualmente se ofertan se identifican 34 en el sitito web: https://www.mexicox.gob.mx/

El sitio MOOC.org (2021) promueve los MOOCs para empresas, entrenamiento y capacitación corporativa; señala que actualmente no se cuestiona si existen razones para implementar programas de capacitación o bien el e-learning, sino la forma en que debe de hacerse; hace énfasis en que, tanto el tiempo del que disponen los empleados como los recursos, son limitados y que la atención debe centrarse en cómo maximizar aspectos como: la atención, la efectividad en el aprendizaje, la rapidez en el desarrollo de las habilidades críticas. Las empresas, para ello, recurren al aprendizaje en línea pues su diseño flexible permite que sea adaptable a empleados de tiempo completo. Subraya que el entrenamiento digital se requiere en todos los sectores, que la tecnología digital es aplicable a todo el ámbito de las organizaciones y que además éstas demandan habilidades digitales en incesante crecimiento. Los MOOCs representan una oportunidad para que los empleados continúen con su educación y preparación; se menciona que "de acuerdo a un informe de Burning Glass Technologies, menos de 1 de cada 5 personas en busca de empleo tienen antecedentes o la experiencia necesarios para igualar lo que buscan estas organizaciones en crecimiento".

La plataforma edX (2021): "es un destino de aprendizaje en línea sin fines de lucro y una plataforma de MOOCs fundada por la Universidad de Harvard y el Instituto de Tecnología de Massachusetts en 2012". Señala contar con la participación de 160 universidades miembro, 350 programas, 3367 cursos y 489,013 personas registradas. En su versión para negocios, se promueve como soluciones de eLearning para impulsar el crecimiento de las empresas a través de entrenamiento y capacitación para el capital humano; afirma que para ello emplea contenidos de alta calidad con el respaldo de las mejores instituciones. De acuerdo con la información que proporciona, sobresalen cuatro grandes áreas temáticas en las que la demanda es alta y el talento es escaso: ciencias de la computación, análisis de datos, habilidades profesionales y administración de empresas; en ellas destacan programas específicos como algoritmos, lenguajes de programación, diseño web y móvil; inteligencia artificial, big data, ciencia de datos Excel; visualización de datos, habilidades blandas, liderazgo inclusivo y estrategia digital, economía, finanzas, entre otros.

El cambio tecnológico se ha generado con tal velocidad en los últimos 20 años, que aquello que se pudo considerar como un concepto revolucionario, como lo fue el término de e-learning apenas en el 2018 ya se considera en desuso, Orri (2018) afirma: "el aprendizaje será digital o no será, por lo que más que de educación, debemos empezar a hablar de e-ducación . . . lo datos muestran que la formación online es la opción prioritaria y mayoritaria "; según consultoras como Crossknowledge, Féfaur e Ipsos, la formación online a través de los MOOC, es el método para la capacitación de empleados que ya está siendo utilizado por alrededor del $70 \%$ de las grandes empresas europeas. Esta formación se ha fortalecido con la eficacia y la flexibilidad de dichas propuestas digitales, que además de contar con las características ya señaladas de ser cursos gratuitos y abiertos, se consideran otras que las vuelven más atractivas; como lo es el hecho de que, transmiten conocimiento con dosis audiovisuales a través de videos cortos, además de la formación de comunidades sociales las cuales promueven el aprendizaje colaborativo y que las plataformas se presentan al usuario de forma llamativa e intuitiva. El dato numérico de más de 78 millones de estudiantes, que alrededor del mundo aprenden de uno de los 9,400 cursos que se ofertan en una diversidad de plataformas especializadas; parece ser el mejor referente de éxito de los MOOC.

De acuerdo con Orri (2018) en relación con el ensamble generado por los MOOC en las empresas, señala que en ese entorno son 
concretados los programas por una amplia mayoría; refiere un porcentaje de $80 \%$ de cumplimiento o finalización. De forma particular cuentan con estudios que indican una preferencia de un $75 \%$ por parte de los millennials, hacia los MOOC como programas de capacitación. DoceboLMS (que es un sistema para la gestión del aprendizaje) arrojó cifras en el 2015 de hasta 35 millones de estudiantes, como una clara aceptación de los programas laborales a nivel mundial.

\section{MÉTODO}

El objetivo sobre el cual se centra el presente estudio, es determinar si los estudiantes de maestría de una universidad pública del noreste de México se están capacitando o no en herramientas digitales.

La ruta seleccionada es cuantitativa y el alcance del estudio es de tipo exploratorio-descriptivo, ya que de acuerdo con Hernández y Mendoza (2018) un estudio puede ser básicamente exploratorio, ya que es flexible en su metodología y se puede utilizar cuando se da inicio para conocer un determinado entorno, lo que permite definir inicialmente cuáles serán los aspectos potenciales para ser considerados y presentar a su vez elementos descriptivos; ya que ellos permitirán especificar ciertas propiedades para un determinado contexto, y de esa forma medir o recabar información interesante para su análisis. En esta investigación no se establece hipótesis de trabajo, ya que en el proceso a seguir no se considera el determinar cómo se relacionan los conceptos o variables y no se aplican predicciones de ninguna índole, solo se enfoca en medir los conceptos. Para dar respuesta a las preguntas de esta investigación, el tipo de diseño a seguir es no experimental de tipo transeccional.

Una vez definida la ruta, el alcance y el diseño de la investigación se selecciona la muestra, la cual se determina como no probabilística por conveniencia. El objeto de estudio son los estudiantes de maestría de una universidad pública del noreste de México que participaran de forma voluntaria en un curso sobre tecnologías y de los cuales es importante conocer su situación laboral actual, además de aspectos iniciales que permitan a los organizadores del curso el poder definir, administrar $y$ tomar decisiones concernientes al desarrollo del curso. La muestra consta de 550 estudiantes.

Para el proceso de recolección de los datos se diseña y aplica a los estudiantes un instrumento de medición tipo cuestionario, se esquematiza una encuesta integrada por seis secciones: datos generales, estudios, situación laboral, capacitación, conocimiento sobre herramientas básicas digitales y los MOOC como herramienta de capacitación; con un total de 3 preguntas con formato abierto y 16 preguntas cerradas (las primeras son analizadas y codificadas para integrarse como items). La encuesta se crea y aplica por medio de la herramienta Formularios de Google de la cual, una vez recopilada la información, se descarga un archivo de Excel mismo que se procesa y registra en la herramienta IBM SPSS (Statistical Package for the Social Sciences) para su análisis final y así conseguir dar respuesta a las preguntas que integran esta investigación. Se realiza una prueba de confiabilidad del instrumento obteniendo un 0.814 de acuerdo con el Alfa de Cronbach, clasificándolo como satisfactorio.

\section{RESULTADOS}

Se aplicó la encuesta a un grupo de estudiantes de los cuales el $51 \%$ son de género femenino y el $49 \%$ de género masculino. La mayoría de los estudiantes están en un rango de edad de 21 a 30 años (55.5\%), un $36.6 \%$ tiene entre 31 y 45 años y únicamente un $8.1 \%$ tienen edad de 46 años o más, como se muestra en la tabla 1 :

Tabla 1. Rango de edad de estudiantes que

\begin{tabular}{ccc}
\multicolumn{3}{c}{ participaron en la encuesta } \\
\hline Edad & Estudiantes & $\%$ \\
\hline $\mathbf{2 1 - 2 5}$ & 118 & 21.5 \\
\hline $\mathbf{2 6 - 3 0}$ & 187 & 34.0 \\
\hline $\mathbf{3 1 - 3 5}$ & 89 & 16.2 \\
\hline $\mathbf{3 6 - 4 0}$ & 66 & 12.0 \\
\hline $\mathbf{4 1 - 4 5}$ & 46 & 8.4 \\
\hline $\mathbf{4 6 - 5 0}$ & 30 & 5.5 \\
\hline
\end{tabular}




\begin{tabular}{ccc}
\hline $\mathbf{5 1 - 5 5}$ & 10 & 1.8 \\
\hline $\mathbf{5 6 - 6 0}$ & 2 & 0.4 \\
\hline $\mathbf{6 1}$ o mas & 2 & 0.4 \\
\hline Total & $\mathbf{5 5 0}$ & $\mathbf{1 0 0 . 0}$ \\
\hline \multicolumn{3}{c}{ Fuente: Elaboración propia. }
\end{tabular}

El grupo de estudiantes encuestados tienen una carrera profesional diversa en el área de las Ciencias Sociales, Políticas, de la Salud, Administración Pública, Relaciones Internacionales, Comunicación, Derecho, Economía, Administración, Contaduría, Ingenierías (Civil, Extractiva, Metalúrgica, Computación, Informática, Eléctrica, Electrónica, Mecánica, Industrial, Transportes, Aeronáutica), Educación, Matemáticas, Física y Química, entre otras.

El $19.1 \%$ de los estudiantes obtuvieron su último título entre el 2001 al 2010, un $70.1 \%$ entre el 2011 al 2020 y sólo el $10.7 \%$ terminaron su carrera antes del año 2000. Además, el $65 \%$ de los estudiantes manifiestan ser autodidactas, un $33 \%$ más o menos y un $2 \%$ no lo es.

Respecto a su estado laboral actual, el $32.2 \%$ de los estudiantes señalan tener un trabajo denominado como home office (trabajo desde casa), el 54.7\%, cuentan con un trabajo que requiere la presencialidad, el $6.5 \%$ por el momento no está trabajando y el $6.5 \%$ refiere haber sido cesado de su centro laboral por la pandemia generalizada por Covid-19. El área laboral en la que se desempeñan actualmente los estudiantes es variada, algunos están en el área Contable, o en Calidad, en el área Comercial, Finanzas, Salud, en Sistemas Computacionales, en el área Legal, Administración, en Construcción, en Educación e Ingeniería.

Las empresas en las que laboran son principalmente del giro de servicios con el $33.1 \%$, siendo uno de los más cambiantes y flexibles porque ayudan a satisfacer necesidades muy variadas. Le sigue el giro industrial con el $29.8 \%$, son empresas que se dedican a crear productos con valor añadido a partir del procesamiento de materias primas, el $16.2 \%$ de las empresas tienen un giro Comercial, es decir, se dedican a la compraventa de productos y el $9.8 \%$ participa en el sector de la Educación (ver la tabla 2). El $10.9 \%$ son micro empresas (menos de 10 empleados), un $12.9 \%$ son empresas pequeñas (menos de 50 empleados), el $17.6 \%$ son empresas medianas (menos de 250 empleados) y el $47.6 \%$ de las empresas son grandes (más de 250 empleados).

\begin{tabular}{|c|c|c|}
\hline Giro & Estudiantes & $\%$ \\
\hline Comercial & 89 & 16.2 \\
\hline Industrial & 164 & 29.8 \\
\hline Servicios & 182 & 33.1 \\
\hline Educación & 54 & 9.8 \\
\hline No labora & 61 & 11.1 \\
\hline Total & 550 & 100.0 \\
\hline
\end{tabular}

Para un $40.4 \%$ de los estudiantes la capacitación es financiada por la Empresa donde labora, y el $12.4 \%$ de los alumnos pagan una parte y la empresa en la que trabajan financia otra parte; mientras un $33.8 \%$ de ellos costean los cursos o talleres con sus propios ingresos.

Las herramientas digitales en las cuales se han capacitado los estudiantes son: el $30 \%$ en Microsoft Office (Excel, Word, PowerPoint), el $28.2 \%$ en Paquetes especializados (SPSS, Minitab, Contpaqi, Aspel SAE, Atlas TI, AutoCad, Banca electrónica, Autodesk Revit 2018, Cisco Routing and Switching, Precore, ERP SAP, ACL Analytics, ErgoIBV, Edivolt, Verint, VWware, Photoshop, Power BI y Meetup en otras), el 3.6\% en Software de Programación o Lenguajes de programación (Oracle, Process Simulador, programación visual Basic, Python, R studio, Studio 5000, Twinmotion, etc), el $24 \%$ se habilitan en el uso de plataformas e internet (Facebook Ads, Finder, Skype, Google meet, classroom, red Rhino, Microsoft Teams, Edmodo, Zoom, Nexus, Webex, Canvas, unity, Odoo) y el $14.2 \%$ de los estudiantes no han recibido preparación alguna, o no señalaron alguna herramienta en la cual se hayan capacitado.

En la tabla 3 se observa que el $23.1 \%$ de los estudiantes no recibieron ningún curso antes de la pandemia o en el año 2019 (esta 
categoría incluye los estudiantes que laboran y no se capacitan, así como los que no laboran y no se capacitan), el $32.2 \%$ obtuvo solamente una capacitación, el $23.6 \%$ de los alumnos realizaron dos cursos, el $7.6 \%, 3$ capacitaciones; $4.9 \%$ ha adquirido 4 capacitaciones y el $8.5 \%$ realizó 5 o más en este periodo. En la tabla 4, se presenta que el $47.3 \%$ de los estudiantes no tuvieron participación en capacitaciones en el año 2020. Además, se observa que el $24 \%$ de los estudiantes realizaron un curso, $10 \%$ tomaron 2 capacitaciones, $6.7 \%$ participaron en 3 cursos, $3.1 \%$ realizaron 4 cursos y el $8.9 \% 5$ o más.

Tabla 3. Cantidad de cursos de capacitación en el año 2019.

\begin{tabular}{ccc}
\multicolumn{3}{c}{ el año 2019.} \\
$\begin{array}{c}\text { Capacitaciones } \\
\text { Sin Pandemia }\end{array}$ & Estudiantes & $\%$ \\
\hline $\mathbf{0}$ & 127 & 23.1 \\
\hline $\mathbf{1}$ & 177 & 32.2 \\
\hline $\mathbf{2}$ & 130 & 23.6 \\
\hline $\mathbf{3}$ & 42 & 7.6 \\
\hline $\mathbf{4}$ & 27 & 4.9 \\
\hline $\mathbf{5}$ o más & 47 & 8.5 \\
\hline Total & 550 & 100.0 \\
\hline \multicolumn{3}{c}{ Fuente: Elaboración propia. }
\end{tabular}

Tabla 4. Cantidad de cursos de capacitación en el año 2020.

\begin{tabular}{ccc}
\hline $\begin{array}{c}\text { Capacitaciones con } \\
\text { pandemia (2020) }\end{array}$ & Estudiantes & $\%$ \\
\hline $\mathbf{0}$ & 260 & 47.3 \\
\hline $\mathbf{1}$ & 132 & 24.0 \\
\hline $\mathbf{2}$ & 55 & 10.0 \\
\hline $\mathbf{3}$ & 37 & 6.7 \\
\hline $\mathbf{4}$ & 17 & 3.1 \\
\hline $\mathbf{5}$ o más & 49 & 8.9 \\
\hline Total & 550 & 100.0 \\
\hline \multicolumn{2}{c}{ Fuente: Elaboración propia. } \\
\hline
\end{tabular}

Como resultado del análisis, respecto a la información sobre la capacitación de acuerdo con el giro de la empresa donde laboran los estudiantes, (ver Tabla 5) se obtiene que son los trabajadores de Servicios ( $18.5 \%$ y $26.2 \%$, antes y durante la pandemia, respectivamente) y de la Industria $(14.5 \%$ y
$22.5 \%$ antes y durante la pandemia, respectivamente) los que más se capacitan en herramientas digitales (al menos tienen una capacitación), los estudiantes que laboran en sector comercial presenta un $22.5 \%$ de capacitaciones durante la pandemia y un $8.5 \%$ sin pandemia y es en el giro de Educación donde menor cantidad de estudiantes se capacitan. (6.4\% y $12.9 \%$, antes y durante la pandemia, respectivamente). Además. los estudiantes que no presentan capacitaciones antes de la pandemia es un $23.1 \%$ y con la pandemia es el $47.3 \%$.

Tabla 5. Capacitaciones con y sin pandemia por giro de empresa laboral

\begin{tabular}{lcc}
\hline $\begin{array}{l}\text { Giro } \\
\text { empresa }\end{array}$ & $\begin{array}{c}\text { Capacitaciones } \\
\text { sin pandemia }\end{array}$ & $\begin{array}{c}\text { Capacitaciones } \\
\text { con pandemia }\end{array}$ \\
\hline Servicios & $102(18.5 \%)$ & $144(26.2 \%)$ \\
\hline Industrial & $80(14.5 \%)$ & $124(22.5 \%)$ \\
\hline Comercial & $47(8.5 \%)$ & $71(22.5 \%)$ \\
\hline Educación & $35(6.4 \%)$ & $43(12.9 \%)$ \\
\hline No labora & $26(4.7 \%$ & $41(7.8 \%)$ \\
\hline Total & $\mathbf{2 9 0 ( 5 2 . 7 \% )}$ & $\mathbf{4 2 3 ( 7 6 . 9 \% )}$ \\
\hline \multicolumn{3}{c}{ Fuente: Elaboración propia. }
\end{tabular}

Al considerar la pregunta de investigación respecto a si las personas de más de 50 años se capacitan, se detecta que el 2.6 son estudiantes que se integran en este rubro y que de éstos el $2.1 \%$ presenta al menos, una capacitación en herramientas digitales antes de la pandemia y durante la pandemia, se observa a un $2 \%$ que se capacitó. El porcentaje de capacitaciones destaca en el rango de edad de 21 a 30 años, considerando que es la edad predominante en nivel maestría.

Respecto a revisar si aún existen trabajadores que tienen niveles de conocimiento bajo en las herramientas básicas de Microsoft Office tales como Word, Excel y PowerPoint; en el análisis de la información se detecta de la herramienta Microsoft Word un $3.1 \%$, de la herramienta Excel un $9.8 \%$ y de PowerPoint un $4.3 \%$ con un grado de conocimiento bajo o muy bajo (ver Tabla 6).

Tabla 6. Grado de conocimiento de los estudiantes sobre las herramientas Microsoft. 


\begin{tabular}{cc}
\hline & $\begin{array}{c}\text { Bajo o } \\
\text { Muy bajo }\end{array}$ \\
\hline Word & 17 \\
\hline$\%$ & 3.1 \\
\hline Excel & 54 \\
\hline$\%$ & 9.8 \\
\hline PowerPoint & 24 \\
\hline$\%$ & 4.3 \\
\hline Fuente: Elaboración propia.
\end{tabular}

Finalmente, en la última sección de la encuesta correspondiente a los MOOC como herramienta de capacitación, se obtiene que solo un $8 \%$ de los estudiantes se han inscrito en un curso en alguna de las plataformas de MOOC; tales como: Coursera, Udemy, EdX, Udacity, Miríada X, Google Actívate, México $\mathrm{X}$, Alison, OpenSAP, o en alguna otra similar. Del porcentaje anterior se detecta que cerca del $3 \%$ han logrado finalizar con éxito al menos un curso.

\section{CONCLUSIONES}

Se puede apreciar en los resultados presentados, que debido a la pandemia, se esperaría que el número de capacitaciones sobre herramientas digitales se incrementara por las nuevas condiciones de trabajo que se dieron con el distanciamiento social; sin embargo, podemos observar (que con la pandemia) que el porcentaje de estudiantes que realizaron al menos una capacitación sobre alguna herramienta digital es de $52.7 \%$, el cual presenta una reducción, comparado con el $76.9 \%$ que se obtuvo sin pandemia. Sobre esto, se muestra también que el $41.5 \%$ reciben una capacitación o ninguna al año, lo cual representa un área de oportunidad para que las empresas puedan capacitar a sus trabajadores o para que las instituciones educativas ofrezcan el apoyo de capacitación a las empresas. Un elemento por considerar para que la capacitación se dé, es contar con el apoyo de la empresa, de esto se detecta que poco más de la mitad de los trabajadores $(52.8 \%)$ son quienes han recibido este beneficio. Se ha mencionado que los MOOC han incursionado como una herramienta de capacitación en línea, en la cual las personas pueden llevar cursos gratuitos o el pagar una módica cantidad para recibir una constancia oficial; en relación a ello, en este estudio se identifica un porcentaje muy bajo de personas que los utilizan y más bajo es aún, la cantidad de personas que han llegado a finalizar con éxito al menos uno de los cursos, a pesar de que el $65 \%$ de los encuestados manifestaron ser autodidactas.

Con respecto a lo anterior, un aspecto a considerar, es que la mayoría de los MOOC o bien los que proceden de universidades $\mathrm{u}$ organizaciones con mayor grado de reconocimiento; sus cursos están en idioma inglés, lo cual puede resultar en un obstáculo para adoptar este medio como capacitación. Por otro lado, es importante considerar sobre éstos, lo que señala el Ing. Christian Hernández Quiroz- Head of Coursera for Campus de Coursera México (UANL, 2021) en su participación en el panel titulado "La alfabetización tecnológica y su impacto en el mundo, a un año de la pandemia"; en este evento, menciona que la pandemia ha originado un incremento en el número de inscripciones a los cursos que ofrecen, siendo antes de la pandemia de 30 millones y ahora casi de 80 millones; sin embargo (comenta en el minuto 58) que las personas que suelen entrar por sí mismas a tomar un curso, sólo el 17\% lo llegan a concluir. De forma particular, este porcentaje aumenta significativamente, cuando se establece una alianza con universidades, corporaciones y gobiernos; de lo cual se presume, que se requiere (en cierta forma) de algún tipo de obligatoriedad para que la persona sea capacitada o bien que se establezca como una capacitación formal.

Para finalizar se resalta en este estudio, que hoy en día, no sólo los jóvenes son quienes se capacitan en herramientas digitales y que son las de Microsoft las que predominan como alternativas para capacitarse; asimismo, se identifica que aún existen personas con niveles de conocimiento bajos o muy bajos acerca de ellas. Se hace énfasis en la necesidad de que la capacitación se incremente hacia otras herramientas digitales para satisfacer la necesidad del mercado actual y contar con el 
capital intelectual necesario, de esta forma evitar la migración, así como el aumentar la productividad de los empleados. Además, se les da a conocer a los estudiantes la importancia de la capacitación y las plataformas que pueden utilizar para llevar a cabo esto. Se les señala que la educación, no termina cuando se gradúan, sino que es un proceso constante que hay que seguir para mantenerse actualizado. 


\section{REFERENCIAS}

America Learning \& Media: Tendencia-Innovación-Tecnología-Cultura. ( Agosto 2016) Informe especial sobre MOOCs de America Learning Media. 32 Plataformas y Proveedores MOOC. Edición 47. http://www.americalearningmedia.com/edicion-047/526-noticias/7340-informeespecial-mooc-de-america-learning-media

Constitución Política de los EstadosUnidos Mexicanos. (17 de mayo de 2021). Título sexto:Del $\begin{array}{llllll}\text { Trabajo } & y & d e & \text { la } & \text { Previsión } & \text { Social. }\end{array}$ http://www.diputados.gob.mx/LeyesBiblio/pdf/1_110321.pdf

Chiavenato, I. (2019). Administración de recursos humanos: El capital humano en las organizaciones. McGraW-Hill

Chiavenato, I.(2020). Gestión del talento humano: El nuevo papel del recurso humano en las organizaciones. McGraw-Hill.

Edx. (2021). Cursos en línea de Harvard, MIT y más. Los estudiantes inquietos cambian al mundo. https://www.edx.org/

EFE noticias.PR Newswire.(4 de abril 2019). 6 de cada 10 empresas en México no invierte en la capacitación de sus empleados: Reporte Laboral Mexico 2019 https://www.efe.com/efe/america/comunicados/6-de-cada-10-empresas-en-mexico-noinvierte-la-capacitacion-sus-empleados-reporte-laboral-2019/20004010-TEXTOE_25680287

El financiero. (2019). Firmas 'cazatalentos'

batallan para cubrir plazas especializadas.

https://www.elfinanciero.com.mx/empresas/firmas-cazatalentos-batallan-para-cubrir-plazasespecializadas/

El universal. (29 de septiembre 2020). El 52\%

de las compañías en México reportan una escasez de talento. https://www.eluniversal.com.mx/cartera/el-52-de-las-companias-en-mexico-reportan-unaescasez-de-talento

Forbes Staff. (10 de enero de 2020). Solo $11 \%$ de las empresas dieron capacitación a empleados en 2019: OCCMundial https://www.forbes.com.mx/solo-11-de-las-empresas-dieroncapacitacion-a-empleados-en-2019-occmundial/

Gestion.Org. (2021). MOOC: nueva modalidad formativa también para empresas. https://www.gestion.org/mooc-nueva-modalidad-formativa-tambien-paraempresas/\#: :text=MOOC\%20son\%20 cursos\%20online\%20masivos,a\%20un\%20p\%C3\%B Ablico\%20m\%C3\%A1s\%20amplio

Gobierno de México. Secretaría del Trabajo y Previsión Social. Concertación y capacitación laboral. https://productividadlaboral.stps.gob.mx/\#

Hays. (2019). Reporte laboral México 2019.

https://www.hays.com.br/documents/63221/3035622/Mexico+SG+2019.pdf

Hernández Sampieri, R., \& Mendoza Torres, C. P. (2018). Metodología de la investigación: Las rutas cuantitativa, cualitativa y mixta. En R. Hernández Sampieri, \& C. P. Mendoza Torres, Metodología de la investigación: Las rutas cuantitativa, cualitativa y mixta. México: Mc Graw Hill.

ILO. (26 de Febrero de 2021). International Labour Organization. https://www.ilo.org/global/topics/skills-knowledge-and-employability/lang--en/index.htm

La mar de llibres. Blog Biblioteca UJI.

(2021). Una aproximación $\quad$ a $\quad$ los MOOC. http://blogs.uji.es/bibliotecauji/mooc/\#: :text=Los\%20Moocs\%20tienen\%20una\%20breve,qu e\%20siguieron\%20casi\%202300\%20estudiantes.

Ley Federal del Trabajo. (23 de abril de 2021).

De la Productividad, Formación y Capacitación de los Trabajadores (pag.43) https://www.senado.gob.mx/comisiones/desarrollo_social/docs/marco/Ley_FT.pdf

Manpower Group. (2020). Manpower Group 
2020 Escasez de talento 2020.

https://www.manpowergroup.com.mx/wps/wcm/connect/manpowergroup/1de44e05-bac6-4c27b8b4-

ef5d1ef3ba9d/2019_InfografIa_escasez_talento_MX.pdf?MOD=AJPERES\&CONVERT_TO $=$ url\&CACHEID=ROOTWORKSPACE.Z18_2802IK01OORA70QUFIPQ192H311de44e05-bac6-4c27-b8b4-ef5d1ef3ba9d-m.PGcTN

MOOC.org. (2021). MOOCs para empresas,

entrenamiento y capacitación

corporativas. https://www.mooc.org/es/corporate-learning

Orri, X. (febrero 2018). Equipos \& talento.

Los MOOC como motor de

transformación social en la empresa.

https://www.equiposytalento.com/tribunas/homuork/los-mooc-como-motor-de-transformacionsocial-en-la-empresa

Redaccion Pymempresario. (4 de abril de

2019). 4 razones para invertir en la

capacitación laboral.

https://www.pymempresario.com/2019/04/4-razones-para-invertir-en-la-capacitacion-laboral/

UANL.(2021). PANEL: "La alfabetización

tecnológica y su impacto en el mundo, a un año de la

pandemia". https://www.youtube.com/watch?v=sQtkah-UiUc

Werther, W.B., Davis, K. y Guzmán Brito,

M.P. (2019). Administración del capital humano. McGraw-Hill 